Preclinical challenge studies of AIDS vaccines in non-human primates have an important role in the AIDS vaccine development effort. However, to be most useful, challenge models need to be improved to more closely reflect the actual biological circumstances of HIV-1 infection and transmission in humans.

\title{
AIDS vaccine models: Challenging challenge viruses
}

Animal models are essential in the search for a vaccine to prevent HIV-1 infection. Of these, rhesus macaque models are, by far, the most important. Rhesus monkeys can be experimentally infected with various simian immunodeficiency virus (SIV) strains of differing virulence, many of which cause simian AIDS (refs. 1,2). However, because HIV-1 does not productively infect macaques, it cannot be used as a challenge virus to assess whether a given vaccine can prevent or ameliorate infection $^{1,2}$. Hence, preclinical AIDS vaccine models rarely test the identical vaccine constructs that are planned for human use. Instead, studies in rhesus macaques explore the potential protective efficacy of vaccine concepts, not the actual vaccines being developed for human trials. To increase the relevance of the macaque model to human vaccine trials, hybrid viruses that contain elements of both the SIV and HIV-1 genomes, designated SHIVs (simian/human immunodeficiency viruses), were first engineered several years ago for use as challenge viruses in pre-clinical AIDS vaccine studies $^{3-6}$. In these chimeric viruses, several HIV-1 genes, most commonly tat, rev and env, are substituted for their SIV homologs. One highly virulent SHIV challenge virus, SHIV 89.6P, has been widely used in vaccine experiments, and is usually now considered the 'virus-of-choice' ${ }^{6,7}$.

Infection of naive macaques with SHIV-89.6P leads, almost invariably, to a rapid and virtually complete loss of $\mathrm{CD}^{+} \mathrm{T}$-cells from the peripheral blood and lymphatic tissues $^{6,7}$ (Fig. 1). Virus replication proceeds at a rapid and highly reproducible rate, and infected macaques progress very rapidly to AIDS and death. The consistency and rapidity of the virological and immunological outcomes of SHIV89.6P challenge is useful in vaccine challenge studies, as a statistically significant conclusion can be drawn from a limited number of animals in an acceptably short time-frame ${ }^{7}$. Furthermore, the extreme virulence of SHIV-89.6P infection has been thought to provide a rigorous challenge model for the comparative evaluation of different vaccine concepts. However, SHIV-89.6P differs in important ways from the HIV-1 strains that most commonly infect and are transmitted between humans. In addition, accumulating evidence suggests that despite its exceptional virulence in macaques, SHIV-89.6P may be a paradoxically easy virus for the immune system to control. In other words, SHIV-89.6P may be a sheep in wolf's clothing, with the potential to lead the AIDS vaccine development effort down the wrong path.

The first SHIVs were constructed when the goal of vaccine development was the outright prevention of HIV-1 infection ('sterilizing immunity') via vaccine-induced neutralizing antibodies against HIV-1 Env. Although vaccines able to induce effective neutralizing antibody responses have not yet been developed, env is still the primary HIV-1 gene included in SHIVs (refs. 1-6). Perhaps by force of habit, this has remained so even when the vaccine approaches being evaluated do not include HIV-1 Env, and are often predicated on the induction of antiviral $\mathrm{CD}^{+} \mathrm{T}$-cell responses rather than neutralizing antibodies. The belief that sterilizing immunity to HIV-1 is an attainable goal has waned recently, and the goal of vaccine development has shifted to the containment of virus replication post-infection (primarily by vaccination-induced antiviral $\mathrm{CD}^{+} \mathrm{T}$-cell responses $)^{8,9}$. A vaccine that allows a substantial containment of viremia in vaccinated individuals who become infected would represent a significant advance, as diminished virus replication is associated with substantially decreased rates of disease progression and HIV-1 transmission ${ }^{10,11}$.

The utility of the SHIV models is obvious, yet their limitations must also be considered. Some limitations are inherent to all animal models of human infections; there may be species-specific, overt or subtle differences in how retroviruses are transmitted, or in the way they cause disease ${ }^{1,2}$. However, other limitations can be specific to certain challenge viruses. For instance, a virus that replicates very poorly in a host may be misleadingly easy to protect against by vaccination, and is of little value for studies of viral pathogenesis. Conversely, vaccine studies that employ particularly virulent viruses or experimental challenges delivered at high doses via intravenous inoculation may underestimate the potential protective efficacy of some vaccine strategies.

The prototypes of genetically engineered SHIVs usually replicated poorly when first inoculated into macaques, and were non-pathogenic ${ }^{1-6}$. Substantially higher replication and greatly enhanced pathogenicity were then achieved by serial passage of the SHIVs through naive macaques ${ }^{1,2,6,7}$. The resulting SHIV variants, exemplified by SHIV-89.6P, mutated to cause a rapid, nearly complete depletion of the total $\mathrm{CD}^{+}{ }^{+} \mathrm{T}$-cell population in newly infected macaques. The acquired ability to extensively destroy $\mathrm{CD} 4^{+} \mathrm{T}$ cells is associated with an increased membrane-fusing capacity of the envelope glycoproteins ${ }^{12}$.

Many vaccine immunogens, alone or in combination, have now been evaluated in the SHIV-89.6P model, including naked DNA, recombinant viruses, subunit proteins and peptides $^{8,9,13-19}$. Despite the diversity of immunogens, a surprisingly consistent outcome has been seen in multiple studies: the vaccinated animals become persistently infected with SHIV-89.6P, but the rapid and complete decline in peripheral $\mathrm{CD}^{+} \mathrm{T}$ cells does not occur ${ }^{8,9,13-19}$. Instead, the animals maintain near-normal $\mathrm{CD}^{+}{ }^{+} \mathrm{T}$-cell counts, and have greatly reduced viremia compared to unvaccinated controls. These results have been greeted with substantial enthusiasm, because the 'successfully' vaccinated animals can control virus replication at levels that are, in HIV-1-infected 
humans, associated with significantly lowered disease progression and transmission rates-probably the most attainable near-term goals of AIDS vaccine research ${ }^{8-11}$.

In HIV-1-infected humans, a disease course like that following SHIV-89.6P challenge of macaques is not unprecedented, but is extremely $\operatorname{rare}^{20,21}$ (Fig. 1). A fear, therefore, is that because the rapid destruction of $\mathrm{CD}^{+} \mathrm{T}$-cells caused by SHIV-89.6P is abnormal, so might be the nature of the protection achieved against this decline induced by such a variety of vaccine antigens.

We have three interrelated concerns about whether SHIV89.6P, despite its logistical advantages, should be considered as the virus-of-choice in pre-clinical AIDS vaccine studies. First, although the HIV-1 env included in SHIV89.6P was derived from a 'dual-tropic' virus that used both the CCR5 and CXCR4 coreceptors for entry into macaque $\mathrm{CD}^{+} \mathrm{T}$-cells, sequential passage in macaques has selected for a SHIV that uses only CXCR4; SHIV-89.6P is a 'pure X4 virus $^{\prime 22}$. Primary infection of humans with viruses that use CXCR4, either alone or as well as CCR5, is rare, but when this occurs, a rapid, irreversible loss of peripheral $\mathrm{CD} 4^{+} \mathrm{T}$ cells is observed ${ }^{23}$. An accelerated loss of $\mathrm{CD}^{+} \mathrm{T}$ cells also ensues whenever CXCR4-using viruses emerge during chronic HIV-1 infection, probably due to their enhanced membrane-fusing capacity and their tropism expansion to target naive $\mathrm{CD}^{+}{ }^{+} \mathrm{T}$-cells in the thymus and periphery ${ }^{23,24}$. However, most HIV-1 strains transmitted between humans use only CCR5 as their coreceptor (R5 viruses), and they do not cause a rapid, irreversible loss of peripheral $\mathrm{CD}^{+} \mathrm{T}$-cells during primary infection ${ }^{23-25}$. Nor does the latter happen
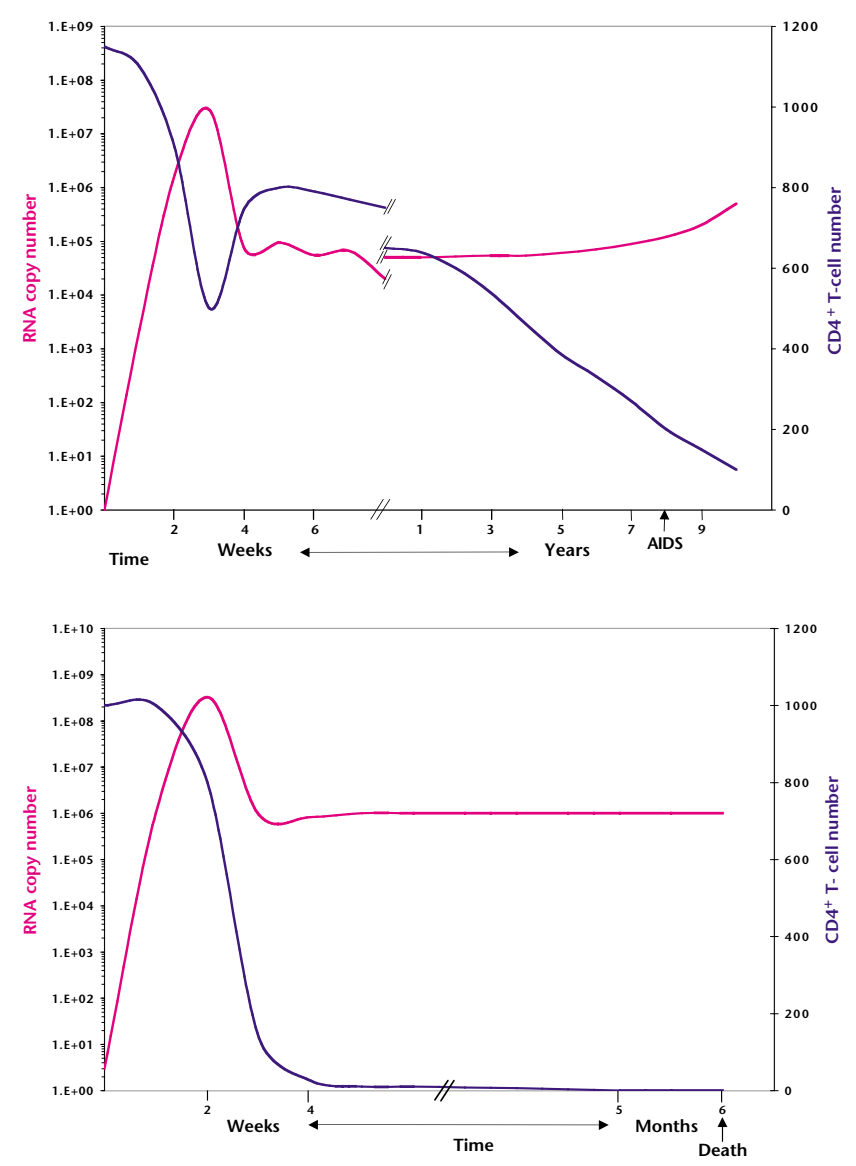

when macaques are infected with an R5 SHIV such as SHIV162P (ref. 26). Overall, X4 viruses and R5 viruses interact with the primate immune system in significantly different ways, yet it is against the transmission and local, early amplification of R5 viruses that an effective HIV-1 vaccine must protect ${ }^{9,25,26}$.

Our second concern relates to the central role of $\mathrm{CD}^{+} \mathrm{T}$ helper cells in determining the outcome of the initial battle between HIV-1 replication and the immune system ${ }^{27}$. Studies in both humans and macaques show that allowing $\mathrm{CD} 4^{+} \mathrm{T}$-cell help to mature by suppressing viral replication during primary infection can be highly beneficial to the subsequent course of disease $\mathrm{e}^{27-30}$. Conversely, a virus that replicates with unusual rapidity may destroy too many $\mathrm{T}$ helper cells before they can coordinate both antiviral B cell and $\mathrm{CD}^{+} \mathrm{T}$ cell-mediated immunity. Such an outcome has a catastrophic effect on the immune response. Indeed, the loss of T-cell help is so striking that humans and macaques with extremely rapidly progressing HIV-1 or SHIV-89.6P infection fail to seroconvert to viral antigens $s^{6,7,12,20,21}$. The temporal development of partially effective adaptive immunity and the destruction of the helper $\mathrm{T}$ cells that coordinate these responses can be finely balanced ${ }^{30,31}$. A very early, vaccine-elicited memory response to viral antigens might restrict the initial replication of SHIV-89.6P just sufficiently to preserve enough of the $\mathrm{CD}^{+} \mathrm{T}$-helper pool during the early, critical generative stages of the antiviral response. Protection might arise by priming of $\mathrm{CD} 4^{+} \mathrm{T}$ cells to provide help for early production of neutralizing antibodies by $\mathrm{B}$ cells, and/or via activation of antiviral $\mathrm{CD}^{+} \mathrm{T}$ cells to limit early expansion in the number of virus-infected cells. Whatever the actual nature of the 'protective' immune effectors raised by various vaccine approaches, their magnitude would be amplified after infection via exposure to viral antigens produced during challenge virus replication. The surviving CD4 ${ }^{+} \mathrm{T}$-helper cells could then coordinate effector $\mathrm{CD}^{+} \mathrm{T}$-cell and $\mathrm{B}$-cell memory responses that contain virus replication.

Thirdly, and perhaps most importantly, SHIV-89.6P is unusually sensitive to autologous neutralizing antibodies ${ }^{6,32}$. If prior vaccination enables a partial preservation of $\mathrm{CD} 4^{+} \mathrm{T}$ cells following challenge, sufficient help would then be available for B cells to mount an anamnestic neutralizing antibody response directed against the SHIV-89.6P Env. Given the sensitivity of SHIV-89.6P to autologous neutralizing antibodies, it may be relatively easy to suppress virus replication, and thereby protect against the rapid decline in $\mathrm{CD}^{+} \mathrm{T}$ cells and rapid disease progression that typically follows SHIV-89.6P infection. However, this dynamic is not

Fig. 1 Contrasting natural histories of AIDS virus infections. HIV -1 infection of humans results in highly variable rates of disease progression that are associated with set-point levels of virus replication and rates of decline of peripheral $\mathrm{CD} 4^{+} \mathrm{T}$ cells. A schematized typical course of progression to AIDS following initial HIV-1 infection is shown, characterized by chronic intermediate levels of plasma viremia, a steady loss of peripheral $C D 4^{+} \mathrm{T}$ cells, and the development of AIDS after 7-10 years (above). In contrast, SHIV-89.6P infection of rhesus macaques results in near-complete destruction of the peripheral $\mathrm{CD} 4^{+} \mathrm{T}$-cell population within the first weeks of infection, uncontrolled high-level virus replication, and rapid progression to AIDS and death (below). 
characteristic of typical HIV-1 infection and the subsequent immune response in humans. In infected humans, HIV-1specific $\mathrm{CD} 4^{+} \mathrm{T}$ cells are rarely acutely depleted to the extent that antiviral immune responses cannot be generated ${ }^{20,21,24}$. Moreover, most primary HIV-1 isolates resist antibody neutralization, and only rarely can the combined activity of antiviral $\mathrm{CD}^{+}$and $\mathrm{CD}^{+}$T-cell responses durably keep virus replication at low levels ${ }^{10,24,27}$.

We believe some of the vaccines that have protected macaques against SHIV-89.6P infection represent important concepts that should be expedited into clinical trials in humans. But the protection elicited by other vaccines may be artifacts of the SHIV-89.6P model, and irrelevant to protecting humans against HIV-1. Few vaccine strategies have been evaluated for protective efficacy using both SHIV-89.6P and alternative pathogenic SIV challenge models. For those that have, the results obtained in the SHIV-89.6P model have always been the more impressive ${ }^{13,16,33,34}$ (and D. Watkins, pers. comm.). Indeed, marked and durable control of a pathogenic SIV has never been achieved by vaccination, other than by a live-attenuated vaccine $e^{1,2,8,9}$. We therefore suggest that vaccine concepts that have given positive results in the SHIV-89.6P model should be retested against an R5 virus that does not cause an abnormally rapid and profound loss of CD4 ${ }^{+}$T-cells (such as SHIV-162P, SIVsmE660, SIVmac251 or SIVmac239). Furthermore, given the tremendous genetic diversity of HIV-1 worldwide, it is essential that the ability of a given vaccine strategy to protect against genetically distinct virus challenges be rigorously investigated in macaque models. So far, all of the experimental SHIV challenge studies have employed vaccine immunogens that are genetically identical (or nearly so) to the challenge viruses ${ }^{13-18}$. The breadth of protective efficacy that can be engendered by vaccination has yet to be addressed experimentally in macaques, yet a single amino-acid change in Gag is reported to be sufficient to enable SHIV-89.6P to escape from immune containment ${ }^{35}$. Given that all of the SHIVs currently used in vaccine studies employ a 'backbone' of SIV genes derived from a single molecular clone (SIVmac239), it will be necessary to develop immunogens that differ from the challenge virus across the viral genome, and not just in the tat, rev and env genes that represent the HIV-1 component of the SHIV.

Animal models are an essential resource for evaluating the safety and comparative immunogenicity of candidate AIDS vaccine strategies in preparation for Phase I human studies. Some vaccine concepts should be rejected before Phase I human studies begin, if a similar concept is so obviously superior in a macaque model. However, if protection from the acutely pathogenic effects of SHIV-89.6P is the only requirement to move a vaccine concept into Phase I human trials, the evaluation pipeline for AIDS vaccines may be over-burdened by vaccines of widely varying immunogenicity and plausibility.

Animal models cannot determine whether a vaccine will be effective against HIV-1 infection of humans; only Phase III trials in humans can do so. However, challenge experiments in the macaque models can potentially add important insights to those gained in Phase I and II studies in humans, and they should meaningfully inform decisions that will impact many thousands of volunteers and involve many millions of dollars. To be most informative and help- ful, challenge studies in macaques should now seek to resolve the most difficult issues in AIDS vaccine development, and, depending on the nature of the scientific question under evaluation, studies may need to use more than a single challenge virus. Within this context, it is essential that macaque models be improved so as to mimic, as closely as possible, the actual circumstances of HIV-1 infection and transmission in humans.

1. Nathanson, N., Hirsch, V.M. \& Mathieson, B.J. The role of nonhuman primates in the development of an AIDS vaccine. AIDS 13 (suppl. A), S113-S120 (1999).

2. Hirsch, V.M. \& Lifson, J.D. Simian immunodeficiency virus infection of monkeys as a model system for the study of AIDS pathogenesis, treatment, and prevention. Adv. Pharmacol. 49, 437-477 (2000).

3. Li, J.T. et al. Persistent infection of macaques with simian-human immunodeficiency viruses. J. Virol. 69, 7061-7067 (1995).

4. Kuwata, T. et al. Construction of human immunodeficiency virus $1 /$ simian immunodeficiency virus strain mac chimeric viruses having vpr and/or nef of different parental origins and their in vitro and in vivo replication. J. Gen. Virol. 76, 2181-2191 (1995).

5. Joag, S.V. et al. Chimeric simian/human immunodeficiency virus that causes progressive loss of $\mathrm{CD}^{+} \mathrm{T}$ cells and AIDS in pig-tailed macaques. J. Virol. 70, 3189-3197 (1996).

6. Reimann, K.A. et al. A chimeric simian/human immunodeficiency virus expressing a primary patient human immunodeficiency virus type 1 isolate env causes an AIDS-like disease after in vivo passage in rhesus monkeys. J. Virol. 70, 6922-6928 (1996).

7. Parker, R.A, Regan, M.M. \& Reimann, K.A. Variability of viral load in plasma of Rhesus monkeys inoculated with simian immunodeficiency virus or simianhuman immunodeficiency virus: Implications for using nonhuman primate AIDS models to test vaccines and therapeutics. J. Virol. 75, 11234-11238 (2001).

8. Schultz, A.M. \& Bradac, J. The HIV vaccine pipeline, from clinical to phase III. AIDS 15 (suppl. 5), S127-S137 (2001).

9. Mascola, J.R. \& Nabel, G.J. Vaccines for the prevention of HIV-1 disease. Curr. Opin. Immunol. 13, 489-495 (2001).

10. Mellors, J.W. et al. Plasma viral load and $\mathrm{CD}^{+}$lymphocytes as prognostic markers of HIV-1 infection. Ann. Intern. Med. 126, 946-954 (1997).

11. Quinn, T.C. et al. Viral load and heterosexual transmission of human immunodeficiency virus type 1. Rakai Project Study Group. N. Engl. J. Med. 342, 921-929 (2000).

12. Etemad-Moghadam, B. et al. Membrane-fusing capacity of the human immunodeficiency virus envelope proteins determines the efficiency of $C D^{+} \mathrm{T}$-cell depletion in macaques infected by a simian-human immunodeficiency virus. J. Virol. 75, 5646-5655 (2001).

13. Barouch, D.H. et al. Reduction of simian-human immunodeficiency virus $89.6 \mathrm{P}$ viremia in rhesus monkeys by recombinant modified vaccinia virus Ankara vaccination. J. Virol. 75, 5151-5158 (2001).

14. Barouch, D.H. et al. Control of viremia and prevention of clinical AIDS in rhesus monkeys by cytokine-augmented DNA vaccination. Science 290, 486-491 (2000).

15. Amara, R.R. et al. Control of mucosal challenge and prevention of AIDS by a multiprotein DNA/MVA vaccine. Science 292, 69-74 (2001).

16. Shiver, J. et al. Replication-incompetent adenoviral vaccine vector elicits effective anti-immunodeficiency-virus immunity. Nature 415, 331-335 (2002).

17. Rose, N.F. et al. An effective AIDS vaccine based on live attenuated vesicular stomatitis virus recombinants. Cell 106, 539-549 (2001).

18. Matano, T., Kano, M., Nakamura, H., Takeda, A. \& Nagai, Y. Rapid appearance of secondary immune responses and protection from acute CD4 depletion after a highly pathogenic immunodeficiency virus challenge in macaques vaccinated with a DNAprime/Sendai virus vector boost regimen. J. Virol. 75, 11891-11896. (2001).

19. Chen, X. et al. Protection of rhesus macaques against disease progression from pathogenic SHIV-89.6PD by vaccination with phage-displayed HIV-1 epitopes. Nature Med. 7, 1225-1231 (2001).

20. Michael, N.L. et al. Rapid disease progression without seroconversion following primary human immunodeficiency virus type 1 infection-evidence for highly susceptible human hosts. J. Infect. Dis. 175, 1352-1359 (1997).

21. Montagnier, L. et al. Human immunodeficiency virus infection and AIDS in a person with negative serology. J. Infect. Dis. 175, 955-959 (1997).

22. Zhang, Y.-j. et al. Use of inhibitors to evaluate co-receptor usage by simian and simian/human immunodeficiency viruses and human immunodeficiency virus type 2 in primary cells. J. Virol. 74, 6893-6910 (2000).

23. Schuitemaker, H. et al. Biological phenotype of human immunodeficiency virus type 1 clones at different stages of infection: progression of disease is associated with a shift from monocytotropic to T-cell-tropic virus population J. Virol. 66, 1354-1360 (1992).

24. McCune, J.M. The dynamics of $C D 4^{+}$T-cell depletion in HIV disease. Nature 410, 974-979 (2001).

25. Veazey, R.S., Marx, P.A. \& Lackner, A.A. The mucosal immune system: primary target for HIV infection and AIDS. Trends Immunol. 22, 626-633 (2001).

26. Harouse, J.M., Gettie, A., Tan, R.C., Blanchard, J. \& Cheng-Mayer, C. Distinct pathogenic sequela in rhesus macaques infected with CCR5 or CXCR4 utilizing 
SHIVs. Science 284, 816-819 (1999).

27. Rosenberg, E.S. et al. Immune control of HIV-1 after early treatment of acute infection. Nature 407, 523-526 (2000).

28. Lori, F. et al. Control of SIV rebound through structured treatment interruptions during early infection. Science 290, 1591-1593 (2000).

29. Lifson, J.D. et al. Containment of simian immunodeficiency virus infection: cellular immune responses and protection from rechallenge following transient postinoculation antiretroviral treatment. J. Virol. 74, 2584-2593 (2000).

30. Oxenius, A. et al. Variable fate of virus-specific CD4(+) T cells during primary HIV-1 infection. Eur. J. Immunol. 31, 3782-3788 (2001).

31. Blancou, P. et al. The infiltration kinetics of simian immunodeficiency virus-specific T cells drawn to sites of high antigenic stimulation determines local in vivo viral escape. Proc. Natl. Acad. Sci. USA 98, 13237-13242 (2001).

32. Montefiori, D.C. et al. Neutralizing antibodies in sera from macaques infected with chimeric simian-human immunodeficiency virus containing the envelope glycoproteins of either a laboratory-adapted variant or a primary isolate of human immunodeficiency virus. J. Virol. 72, 3427-3431 (1998).

33. Ourmanov, l. et al. Comparative efficacy of recombinant modified vaccinia virus
Ankara expressing simian immunodeficiency virus (SIV) Gag-Pol and/or Env in macaques challenged with pathogenic SIV. J. Virol. 74, 2740-2751 (2000).

34. Seth, A. et al. Immunization with a modified vaccinia virus expressing simian im munodeficiency virus (SIV) Gag-Pol primes for an anamnestic Gag-specific cytotoxic T-lymphocyte response and is associated with reduction of viremia after SIV challenge. J. Virol. 74, 2502-2509 (2000).

35. Barouch, D.H. et al. Eventual AIDS vaccine failure in a rhesus monkey by viral escape from cytotoxic T lymphocytes. Nature 415, 335-339 (2002).

${ }^{1}$ Departments of Medicine and Microbiology \& Immunology and The Emory Vaccine Center, Emory University School of Medicine Atlanta, Georgia, USA

${ }^{2}$ Department of Microbiology and Immunology

Weill Medical College of Cornell University

New York, New York, USA

\section{AUTHOR SUBMISSION GUIDE}

\section{Long Article}

Abstract - 100-150 words of simple prose, no technical details. Focus on general advance and implications. No references.

Main text - (3000 word max) Separate sections for introduction, results, discussion and methods (800 word max). Subheadings for results and methods. Reference max of 45. Display items: 6 max including tables, figures can be multipaneled.

\section{Short Article}

Abstract - One terse paragraph of 100-200 words, with references, providing background, main results and implications. Simple prose, no technical details. This is the only introductory material in a short article.

Main text - Results and discussion (1500 word max) immediately follows the abstract, referencing first display item. Subheadings for methods section only (800 word max). References max 25.

\section{Methods}

- Remove all methodology and discussion from the figure legends. This includes concentrations, incubation times temperatures, and so on.

- Oligonucleotide sequences are written 5'-GATCGACT-3' (note: no spaces between the upper-case nucleotide letters).
- Provide locations of manufacturers, without abbreviations (city and state, for US; city and country for non-US): (Vector, Burlingame, California).

- Units except for \% should be preceded by a space ( $7^{\circ} \mathrm{C} ; 12 \mathrm{mM} ; 55 \%$ ).

- Use symbol font for "micro": mM, not uM. If using Microsoft Word, go to "insert/symbols" to make sure the symbols do not drop out when the font changes.

\section{General Style Points}

- Use the active, not passive voice throughout the text: "We examined the retinas of the mice.", not "The retinas of the mice were examined."

- Abbreviations are not used in abstracts. Spell out abbreviations at first use in body of text.

- Mutants are referred to with superscript as follows: "C1qa+/+" and not "C1qa+/+" or "+/+". Gene designation only is italicized; the designation for 'zygosity' is superscripted and not italicized.

- The "P" for P values is upper-case and italicized.

- The " $\mathrm{n}$ " for number is lower-case and italicized.

- Kilodalton is abbreviated $\mathrm{kDa}$.

- Full postal and email address is required for each author

- We strongly recommend that the article be read by a native English-speaking scientist not directly related to your field of research

- In the text, refer to figures as (Fig. 1a, b and c) or (Table) in parentheses, at end of text referring to figure (not "As shown in Fig. 1, ...")

\section{Display items}

- In the text, refer to figures as (Fig. 1a, b and c) or (Table) in parentheses, at end of text referring to figure (not "As shown in Fig. $1, \ldots$ ")

- "Fig." is abbreviated and followed with a period; "F" is upper-case (Fig.).

- Panel letter should be lower-case, and italicized. - Figures must appear in text in order: $1 \mathrm{a}, 1 \mathrm{~b}, 2 \mathrm{a}$ $2 \mathrm{~b}$

- Tables must be supplied as part of a simple word document, if possible, convert the table to text usings tabs to separate entries.

- Provide a brief title (one sentence of less than 10 words) describing the figure or table as a whole in the figure legend.

- Symbols should be identified in figure caption only; not in the image itself.

For More Information see http://medicine.nature.com 